\title{
ChAngement LinguistiQue ET LeXIQUe-GRAMMAIRE
}

\author{
Michele DE GIOIA \\ Università degli Studi di Padova
}

\begin{abstract}
En): This study focuses on the problem of the relationship between two linguistic research areas: language change and Lexicon-Grammar. For the first area, the linguistic notions of "change" and "variation" and the way they lead to orality are explored. For the second area, the method and specific fundamental principles are formulated; it is then shown how several Lexicon-Grammar descriptions capture interesting phenomena of linguistic change. Finally, after theoretically framing these fields and highlighting the possibilities of contact, the author suggests further perspectives for collaborative research.
\end{abstract}

Résumé (Fr) : Nous avons étudié la problématique du rapport entre les deux domaines de recherche du changement linguistique et du Lexique-Grammaire. Pour le premier domaine, nous avons approfondi les notions linguistiques de « changement » et « variation », et indiqué comment elles débouchent sur l'oralité. Pour le second domaine, nous avons présenté la méthode et précisé certains principes fondamentaux; nous avons ensuite présenté plusieurs descriptions lexicogrammaticales qui enregistrent des phénomènes intéressants de changement linguistique. Enfin, après avoir cadré théoriquement ces domaines et mis en valeur des possibilités de contact, nous avons également suggéré des perspectives de recherche collaborative.

Key-words (En): Language change; Lexicon-Grammar

Mots-clés (Fr) : Changement linguistique ; Lexique-Grammaire

DOI : $10.32725 /$ eer.2021.014

...dans la langue il n'y a que des différences.

Ferdinand de Saussure, $C L G$

En 2015 nous publiions un article d'introduction à la méthode du LexiqueGrammaire (DE GIOIA, 2015), que nous adoptons depuis longtemps en élève de Maurice Gross, son fondateur. Nous nous y adressions plus spécifiquement aux jeunes doctorants et docteurs en sciences du langage, de même que nous visons ici les chercheurs qui œuvrent en sociolinguistique et notamment dans le domaine du changement linguistique.

Notre but ${ }^{1}$ est d'établir un rapport entre changement linguistique et LexiqueGrammaire - notés dorénavant CL et LG -, deux domaines de recherche qui restent à ce jour assez séparés. Nous les illustrons et les cadrons grâce à plusieurs définitions et citations, afin de mettre en lumière l'utilité du LG pour étudier le CL. Nous avons également l'ambition de suggérer quelques pistes de recherche peu explorées et prometteuses, reliant les deux domaines.

\footnotetext{
${ }^{1}$ Nous remercions Éric Laporte (LIGM, Université Gustave Eiffel), ainsi que les évaluateurs anonymes, pour leurs suggestions pertinentes.
} 


\section{Le changement linguistique}

Pour appréhender le terme ainsi que la notion, consultons d'abord deux dictionnaires français spécialisés et réputés. Le premier est La grammaire d'aujourd'hui qui, en dépit du titre principal, porte comme sous-titre Guide alphabétique de linguistique française, par ARRIVÉ et al. (1986). Le second est le Dictionnaire de linguistique, par DuBOIS et al. (2002).

Dans les deux ouvrages, on trouve l'entrée changement, sans l'adjectif linguistique (évidemment, parce qu'on ne chercherait pas changement climatique dans un dictionnaire de linguistique...).

Dans le premier, l'entrée renvoie tout de suite à deux autres adresses :

changement Voir DIACHRONIE et VARIATION. (ARRIVÉ et al., 1986 : 99)

Il s'agit donc d'une définition par renvoi. L'adresse diachronie, qui s'étend sur environ quatre pages (1986:220-224), explique comment

Le changement dans le temps atteint tous les systèmes constitutifs de la langue: phonologique, morphosyntaxique et lexicosémantique. (ARRIVÉ et al., $1986: 220-224$ )

L'adresse variation, qui prend moins d'une demi-page (1986:678), est à la fois succincte et dense. Elle signale une opposition entre variation et changement :

La variation linguistique s'observe dans l'espace ou selon les couches socioculturelles [...] En règle générale, variation, ainsi compris, s'oppose à changement, qui vise les mutations diachroniques. Toutefois, on observe parfois des phénomènes d'échange entre ces deux mots. (ARRIVÉ et al., $1986: 678$ )

Le sens de variation indiqué ci-dessus par ARRIVÉ et al. (1986) est l'usage consensuel en linguistique que nous retenons dans cet article.

Dans le second dictionnaire, l'entrée changement, assez longue (DUBOIS et al., 2002 : 82-83), est composée de trois acceptions. La première contraste un peu avec l'opposition D'ARRIVÉ et al. (1986: 678) car elle rend compte d'une synonymie possible unissant changement et variation:

1. Le changement, appelé aussi variation, est sans doute le caractère le plus important du langage. (DuBOIS et al., $2002: 82$ )

La deuxième acception est totalement consacrée à la problématique des changements phonétiques, qui ont été les premiers à retenir l'attention des sociolinguistes. En revanche, la troisième acception ci-dessous corrèle les notions de changement et de structure que nous allons reprendre plus loin :

3. En grammaire générative, le changement structurel est un des aspects de la transformation consistant, après l'analyse* structurelle, à effectuer diverses opérations de suppression, de réarrangement, etc., sur la structure ainsi analysée. (V. TRANSFORMATION.) (DUBOIS et al., 2002 : 83) 
Citons en passant l'entrée avec adjectif changement linguistique, publiée dans Wikipédia :

Un changement linguistique est la variation phonétique, morphologique, sémantique, syntaxique, ou d'autres caractéristiques, d'une langue dans le temps. [...] Toutes les langues changent en permanence et le font de différentes façons. Marcel Cohen détaille différents types de changements linguistiques dans les deux catégories d'évolutions externes et internes des langues. («Changement linguistique », Wikipédia) ${ }^{2}$

Si l'on passe aux études spécialisées, un article fondamental, consacré aux fondements empiriques d'une théorie du CL, approfondit l'articulation entre CL et variation :

L'article de Weinreich, Labov et Herzog (1968) propose une première articulation entre variation et changement linguistique en considérant la variation comme un phénomène inhérent à toute langue, qui permet de manière centrale la transition entre deux états de langue. (LEDEGEN \& LÉGLISE, 2013 : 402-403)

Cet article à six mains cherche à définir un nouveau modèle théorique :

Les propositions de base pour l'élaboration d'un modèle adéquat et conforme à la diversité des faits d'usage seront alors les suivantes : $1^{\circ}$ la langue est un objet qui possède une hétérogénéité ordonnée et, corollaire, $2^{\circ}$ l'état de langue fonctionne comme déterminant ses propres changements. (LeCOINTRE \& LE GALLIOT, 1973 : 20)

De plus, le même article aborde la réflexion sur les facteurs responsables du CL :

Linguistic change is not to be identified with random drift proceeding from inherent variation in speech. Linguistic change begins when the generalization of a particular alternation in a given subgroup of the speech community assumes direction and takes on the character of orderly differentiation. ${ }^{3}$ (WEINREICH, LABOV \& HERZOG, $1968: 187$ )

La réflexion est approfondie par LABOV, l'un des fondateurs de la sociolinguistique moderne, dans un ouvrage incontournable en trois volumes (1994, 2001, 2010). Le premier porte sur les facteurs internes dans le CL, le deuxième sur les facteurs sociaux, le troisième sur les facteurs cognitifs et culturels. En tout cas,

2 Cf. https://fr.wikipedia.org/w/index.php?title=Changement_linguistique\&oldid=134895509\# Cohen1950 (dernière consultation: 27/03/2020). Cet article de Wikipédia ne renvoie qu'à un seul ouvrage, à savoir un volume de COHEN (1950).

3 « Le changement linguistique ne doit pas être identifié avec une dérive aléatoire résultant d'une variation inhérente de la parole. Le changement linguistique commence lorsque la généralisation d'une alternance particulière dans un sous-groupe donné de la communauté de la parole devient dominante et prend le caractère d'une différenciation ordonnée. » (Notre traduction) «Il cambiamento linguistico non deve essere identificato con una deriva casuale che procede dalla variazione intrinseca del linguaggio. Il cambiamento linguistico inizia quando la generalizzazione di una particolare alternanza in un dato sottogruppo della comunità linguistica diventa dominante e assume il carattere di differenziazione ordinata. » (Notre traduction)

$$
-7-
$$


les citations ci-dessus mettent en lumière un espace sémantique partagé principalement par les termes (parfois synonymiques) $C L$, variation, ainsi que le terme morphologiquement apparenté variété. Pour s'orienter dans cet espace, on peut suivre Gadet lorsqu'elle affirme que les sociolinguistes parlent

de variétés pour désigner différentes façons de parler, de variation pour les phénomènes diversifiés en synchronie, et de changement pour la dynamique en diachronie (GADET, $2003: 7$ )

En ce qui concerne la notion de variation, considérons que :

la variation est une composante essentielle et non un phénomène marginal dans le système linguistique (COMBETTES, $2008: 135)$

Précisons qu'il n'y a pas qu'une seule définition mais que, chez les sociolinguistes, il existe deux manières de concevoir la variation, qui peut être orientée vers le système linguistique ou bien vers le locuteur. Elles sont schématisées synthétiquement par BERRUTO (2017) et adaptées par GADET (2020 : 22-23) :

\begin{tabular}{|l|l|l|}
\hline & Orientation vers le système & \multicolumn{1}{|c|}{ Orientation vers le locuteur } \\
\hline Principe de base & $\begin{array}{l}\text { La variation comme reflet de } \\
\text { l'expression du social }\end{array}$ & $\begin{array}{l}\text { La variation comme produit de } \\
\text { l'activité du locuteur }\end{array}$ \\
\hline $\begin{array}{l}\text { Concepts } \\
\text { fondamentaux }\end{array}$ & $\begin{array}{l}\text { Communauté, corrélations } \\
\text { Variables, variantes } \\
\text { Variétés }\end{array}$ & $\begin{array}{l}\text { Représentations } \\
\text { La parole comme acte d'identité } \\
\text { Choix }\end{array}$ \\
\hline Objectifs & $\begin{array}{l}\text { Décrire les formes et les sens } \\
\text { Concevoir la variation comme } \\
\text { moyen d'assurer les besoins } \\
\text { communicatifs d'une société }\end{array}$ & $\begin{array}{l}\text { Comprendre comment les locuteurs } \\
\text { mettent en ceuvre des ressources } \\
\text { linguistiques pour produire du sens }\end{array}$ \\
\hline Catégories d'analyse & Surtout linguistiques & Sociales, biographiques \\
\hline Perspective & Essentialiste & Constructiviste \\
\hline
\end{tabular}

Fig. 1.

C'est pour cette raison que :

Le but de Weinreich ${ }^{4}$ demeure bien de pouvoir décrire la langue comme un système, mais qui fonctionne non pas en dépit des variations de tous ordres que l'on constate (et l'on parle alors de «moyenne », de changements «négligeables », d' «idéalisation nécessaire ») mais avec ces phénomènes de variation. (DelaveAu et al., $1972: 40$ )

\footnotetext{
${ }^{4}$ En fait, Delaveau et al. (1972: 40) font référence ici à l'article de Weinreich, Labov, HerzoG (1968).
}

$$
-8-
$$


La notion de variation ne saurait être dissociée de celle de norme $e^{5}$, qui est à son tour polysémique car il existe une norme orale, écrite, pédagogique etc. De plus, des notions géminées de variation concernent certaines dimensions du système linguistique, vu qu'on étudie la variation phonologique, lexicale, syntaxique etc. Considérons le numéro thématique de la revue Langue française consacré à $L a$ variation en syntaxe (GADET, 1997). Dans les années 70 et 80 , la notion de variation syntaxique est liée à des discussions sur l'existence de synonymes syntaxiques purs, alors que vers la fin du $\mathrm{XX}^{\mathrm{e}}$ siècle elles se sont poursuivies, à plus grande échelle, dans le cadre de grands corpus linguistiques (BLANCHE-BENVENISTE, 1997). La notion est également cernée par opposition à la variation phonologique et en explorant quelques phénomènes du français parlé (GADET, 1997). Plus récemment, une étude remarquable montre que la notion est un artéfact construit par des approches qui ne reconnaissent pas la présence de règles et de distinctions propres à tel ou tel usage (DEULOFEU, 2017) et - ajoutons-nous - à telle ou telle norme.

Par ailleurs, si les deux notions de variation et $C L$, ainsi que les autres qui leur sont reliées de synchronie et diachronie, sont traditionnellement opposées, elles peuvent être rapprochées via la notion structuraliste de "synchronie dynamique ", largement exploitée par Martinet ${ }^{6}$ mais posée, bien avant lui, par Mathesius ${ }^{7}$. En effet, la théorie de la potentialité chez le linguiste tchèque a mis en lumière qu'on peut découvrir des oscillations dynamiques (ou des fluctuations dynamiques, d'après LAZARD, 2007: 14) aussi en synchronie, c'est-à-dire des variations aléatoires observées dans l'actualisation, en discours, du système synchronique.

Le domaine du CL comporte un tel réseau de notions et de termes reliés qu'il n'est pas possible de les traiter ici dans toute leur extension. En effet, rappelons que notre intention n'est pas celle d'établir un état de l'art complet des théories du CL, mais d'en esquisser un tableau général qui puisse servir de pont entre le CL et le LG. Bref :

La description du changement linguistique concerne tous les niveaux de la langue orale et écrite, tous les styles, tous les locuteurs, et constitue donc une thématique transversale (Claire MARTINOT) $)^{8}$

\footnotetext{
${ }^{5}$ Si ce n'est en sociolinguistique, où on préfère ne pas parler de norme mais de langue standard, ou « légitime » selon la formulation de Bourdieu (ARMStrong, 2008 : 17). Par ailleurs, parler de norme nous fait remonter au moins à Vaugelas.

${ }^{6}$ RADIMSKÝ (2007 : 10) souligne que c'est par MARTINET (1955) que « les structuralistes redécouvrent le concept de la dynamique synchronique du système linguistique. Mais il s'agit là d'une vraie réinvention faite pratiquement ex nihilo ». Encore MARTINET (1975:9) évoquera « une synchronie dynamique, où l'attention se concentre sur un seul et même état, mais sans qu'on renonce jamais à y relever des variations et à y évaluer le caractère progressif ou récessif de chaque trait ».

${ }^{7}$ Nous renvoyons surtout à MATHESIUS (1911) et son célèbre article en tchèque (traduction anglaise dans VACHEK, 1964 : 1-32) sur « la potentialité » du langage, ainsi qu'au numéro thématique 1-2/2007 de l'Écho des études romanes édité par RADIMSKÝ, qui a été entièrement consacré à la question de la synchronie dynamique du système linguistique (https://www.eer.cz/?s=2007_1-2, dernière consultation : 22/07/2021).

${ }^{8}$ Comme l'explique le Descriptif thème transversal $2: 2019-2023$. Linguistique du changement par Claire MARTINOT (Sorbonne Université \& INSPE de l'Académie de Paris).
} 
Le CL est un phénomène multidimensionnel puisqu'il touche les diverses dimensions du système linguistique. Cependant, parmi celles-ci, c'est encore l'oralité qui l'emporte en tant qu'objet privilégié de l'attention des chercheurs. En effet, depuis les années 1970, on assiste à un

changement de paradigme en linguistique contemporaine vers une conceptualisation de la langue avec ses réalisations non seulement à l'écrit, mais aussi à l'oral (WISSNER, 2012 : 249)

L'idée de base est que, pour connaître une langue et son fonctionnement, il est réducteur de s'appuyer sur la grammaire traditionnelle, considérée principalement comme une grammaire de la langue écrite, et souvent même d'un écrit particulier (i.e. littéraire). Par contre, de nouvelles connaissances grammaticales doivent être acquises en tenant compte de la langue dans son ensemble composite de productions écrites et surtout orales. De plus, c'est plutôt dans l'oralité que les phénomènes de variation et de CL peuvent être rencontrés. C'est dans l'oralité qu'on trouve une grammaire tout à fait nouvelle, qui admet par exemple des prononciations et des usages différents, des répétitions, des écarts par rapport à la norme, souvent traités comme des erreurs dans une perspective traditionnelle. Ainsi commence, depuis les années 1970, «la grande vague de l'oral 》 (BLANCHE-BENVENISTE \& JEANJEAN, 1986 : 43-46), en raison de laquelle

nombreux sont les linguistes qui considèrent qu'il est nécessaire d'exploiter des « collections ordonnées d'enregistrements de productions linguistiques orales et multimodales ", communément appelées corpus oraux (déf. d'apr. BAUDE, 2006). (WISSNER, $2012: 249$ )

La volonté grandissante de disposer de corpus $^{9}$ oraux pousse les chercheurs à publier, en 2006, un Guide des bonnes pratiques (BAUDE, 2006) pour la collecte et l'utilisation de ces corpus. En 2008, la revue Verbum consacre un volume entier aux corpus oraux et notamment au recueil et à l'analyse des données (DEBAISIEUX et al., 2008). En 2010, la sociolinguistique est tellement concernée par ces corpus qu'un système est inventé (EXMARaLDA) pour faciliter l'exploitation et la réutilisation des corpus oraux déjà existants (SCHMIDT, 2010). En 2012, une étude contient une liste succincte des corpus, des bases de données et des plateformes en ligne, que l'auteure juge utiles pour mener des recherches sur le lexique diatopiquement marqué du français moderne (WISSNER, 2012 : 262-272). Il s'agit notamment de 40 outils répartis en deux sections : 4 outils dans la section « plates-formes et portails » et 36 dans « corpus et bases de données ». Au total, 17 outils permettent l'exploitation de grands corpus oraux et/ou fondés sur la transcription (comme la transcription des entretiens). 11 outils impliquent également, à partir de leur dénomination, le renvoi à la dimension linguistique de l'oralité. Parmi les 40 outils, on en trouve des historiques (par exemple CLARIN, CNRTL, EUROPRESSE et FRANTEXT), qui ont été conçus pour des recherches variées, y compris littéraires, à partir du XVI ${ }^{\mathrm{e}}$. Tout cela fait ressortir encore plus le

${ }^{9}$ «Le terme corpus est fortement polysémique en sciences du langage » (CAPPEAU \& GADET, 2007 : 101). 
nombre d'outils concernant l'oral. Par ailleurs, tous permettent d'étudier à la fois des observables divers, selon une ouverture méthodologique à caractère ethnosociolinguistique. Il existe aussi d'autres études qui renseignent sur les corpus de français parlé et écrit, où la liste des corpus oraux est plus longue ${ }^{10}$. Ce qui rend manifeste l'importance croissante accordée à la dimension de l'oralité.

Tout compte fait, on constate que le sociolinguiste, de nos jours, explore de plus en plus de grands corpus, mis au point principalement dans le domaine de l'oralité. Cependant, puisque ces corpus sont construits suivant des méthodes et des finalités différentes, leur méconnaissance soulève bien des difficultés dont il faut être conscient lors de leur exploration :

La facilité à organiser les données avec un concordancier favorise un travail sur de gros corpus. Avec une conséquence inattendue : le corpus, dès qu'il dépasse une certaine taille, est rarement connu en profondeur. Ce qui est utilisé se limite souvent à des ensembles de lignes qui sont le produit des requêtes formulées. Le plaidoyer en faveur de données réelles et contextualisées débouche de fait sur une vision fragmentée, partielle, de suites de lignes discontinues. On retrouve alors une coupure que l'on avait voulu croire abolie par les grands corpus. (CAPPEAU \& GADET, $2007: 102$ )

De plus, les corpus ne peuvent être utiles pour tout type de recherche; par exemple,

la syntaxe et le discours ne sont que faiblement impliqués dans la détermination des variétés diastratiques : ainsi, des entités comme la « langue des jeunes » ou le «français populaire » sont identifiables avant tout à travers le lexique, le phonique (segmental et prosodique), ou le morphologique, ou encore par le discursif à travers des formules toutes faites (riche de chez riche) ; mais très peu par la syntaxe. (GADET, $2006: 84$ )

Par conséquent, la complexification apportée par la prise en compte de la diversité sociologique est compensée, dans les grands corpus oraux, par une réduction des ambitions en termes de couverture lexicale et syntaxique. Néanmoins, les deux domaines du lexique et de la syntaxe étant également importants pour étudier le phénomène du CL, le sociolinguiste qui voudrait les analyser en extension nécessiterait d'autres données ou corpus. Nous allons montrer que le LG pourrait lui fournir les informations dont il aurait besoin.

\section{Le Lexique-Grammaire}

Nous avons publié ailleurs (DE GIOIA, 2015) une introduction à la méthode du $\mathrm{LG}$, en illustrant le chemin qui va du structuralisme à la grammaire distributionnelle, puis à la grammaire transformationnelle (i.e. à plusieurs grammaires transformationnelles, dont celles de Harris et de Chomsky), puis au LexiqueGrammaire.

${ }^{10} \mathrm{Cf}$. https://perso.atilf.fr/apotheloz/corpus/ (dernière consultation : 22/07/2021). 
Nous indiquons ce chemin d'une manière plus rapide ici par des citations de DUBOIS et al. (2002). Nous présentons ensuite les traits essentiels du LG et finalement son utilité pour étudier le CL.

[Chez Saussure] Les signes tirent leur valeur des oppositions qu'ils contractent avec les autres signes : un signifiant n'étant constitué que par les différences qui séparent son image acoustique de celles de tous les autres; un signifié ne se définissant pas par son contenu, sa substance, mais négativement par les rapports qu'il entretient avec les autres termes du système. Chaque signe est donc une unité discrète qui définit une combinatoire syntagmatique et paradigmatique à l'intérieur du système formel et structuré qu'est la langue.

$[\ldots]$

Ces hypothèses et ces analyses de F. de Saussure servent de postulats pour les diverses tendances fonctionnalistes et structuralistes du début du XXe siècle jusque vers 1960 : École de Prague, N. S. Troubetskoï, R. Jakobson, A. Martinet.

$[\ldots]$

S'inspirant de L. Bloomfield, les linguistes américains développent, au milieu du $\mathrm{XX}^{\mathrm{e}}$ siècle, sous le nom de distributionnalisme (Z. S. Harris) une théorie du langage plus radicale que le structuralisme européen. Ils partent, en effet, du postulat que la langue en synchronie, ou code, est composée d'unités discrètes : les morphèmes, qui se définissent par les relations syntagmatiques qu'ils entretiennent avec les autres à l'intérieur du système. [...] Il s'agit, par segmentations successives, de mettre au jour des constituants immédiats hiérarchisés, dont la distribution est définie par les environnements. Les éléments dotés d'une distribution identique sont regroupés dans des classes distributionnelles (taxinomie), d'où l'on peut inférer (procédure de découverte) la grammaire de la langue tout entière. Le sens n'est conservé qu'à titre technique comme identification des énoncés. L'analyse distributionnelle est exploitée dans de nombreux travaux (syntaxe, analyse du discours, linguistique quantitative, etc.) et est adoptée par des linguistes européens au début des années 60. (DuBOIS et al., $2002: 286-287$ )

À propos de l'analyse distributionnelle, dans la citation ci-dessus de Dubois, il manque peut-être une reconnaissance de l'idée de HARRIS (1962: 18-19) de décomposer les phrases en parties élémentaires à l'aide de la notion de transformation syntaxique, et d'obtenir comme parties élémentaires des phrases élémentaires et divers autres segments.

Enfin le LG vint... car c'est au cours des années 1960 que Maurice GROSS ${ }^{11}$, élève de Zellig S. Harris, publie le premier volume de sa grammaire transformationnelle du français, consacré à la Syntaxe du verbe (1968). Suivront deux autres volumes sur la Syntaxe du nom (1977) et sur la Syntaxe de l'adverbe (1986a), ainsi que «la Bible» des lexico-grammairiens, à savoir Méthodes en syntaxe (1975). Le cadre théorique tire son origine de la grammaire distributionnelle puis transformationnelle de HARRIS $(1976,2007)$, fondée sur des représentations syntaxiques. Celles-ci donnent lieu à des travaux expérimentaux qui testent, confirment et peaufinent ces représentations, au moyen d'opérations de transformations permettant aux linguistes «de découvrir et de localiser les contraintes syntaxiques (et sémantiques) qui lient les éléments des phrases. » (GROss, 1975 : 9) Mais alors que Harris ne prend que des exemples de mots et de constructions, en LG ces expériences deviennent systématiques pour tout le vocabulaire et toutes les constructions syntaxiques pour lesquelles les entrées

${ }^{11}$ Sa première publication paraît en 1963, d'après la Maurice Gross' Bibliography : 1963-2006 en ligne : http://infolingu.univ-mlv.fr/ (dernière consultation : 22/07/2021). 
lexicales se comportent différemment les unes des autres. Le LG traite deux entrées lexicales d'un même mot (compter sur Marie, compter les calories) exactement comme si c'étaient deux entrées lexicales morphologiquement distinctes (convoquer Marie, enchaîner les arguments), sans chercher à représenter le lien psycholinguistique. L'innovation - et donc aussi l'apport historique - du LG réside justement dans son ambition d'aborder systématiquement et dans toute sa complexité l'articulation du vocabulaire avec la syntaxe.

Le principe fondamental du LG est que «l'unité significative du lexique est la phrase simple » (GROSS, 1986b : 299), et non pas le mot qui n'a aucune autonomie syntactico-sémantique en dehors de la phrase. Autrement dit, l'unité significative du lexique est une séquence phrastique linéaire d'éléments linguistiques combinés entre eux par des propriétés syntactico-sémantiques. Par conséquent, la phrase simple est le lieu d'intersection du lexique et de la grammaire.

Pourquoi décrire systématiquement les phrases simples ? Parce qu'elles sont les unités minimales de sens et qu'elles diffèrent les unes des autres par leurs propriétés syntactico-sémantiques. Les descriptions prennent la forme de tables syntaxiques où des phrases simples en entrées sont croisées avec des propriétés syntacticosémantiques. L'ensemble des tables relatives à une langue prend lui aussi le nom de LG. Ainsi, la dénomination de LG s'applique en même temps à une méthode de description linguistique et aux résultats de la description, sous forme de tables syntaxiques.

Voici un extrait de la table 16 des verbes à constructions complétives du français (GROSS, $1975: 380)$ :

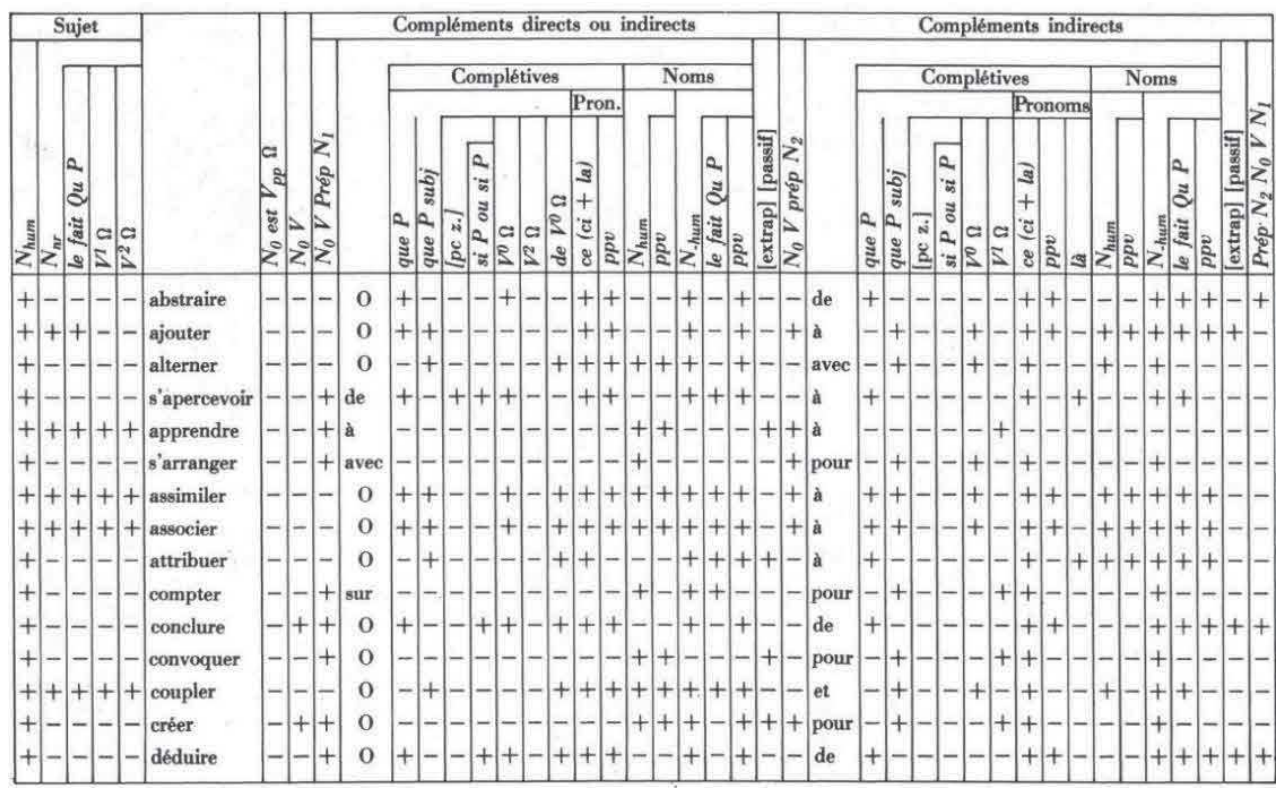

Fig. 2.

Le LG ambitionne d'aborder systématiquement et dans toute sa complexité l'articulation du vocabulaire avec la syntaxe, selon une méthode de description des $-13-$ 
faits de langue qui comporte l'attribution de propriétés aux diverses entrées lexicales. Le LG est donc une représentation discrète, un vrai modèle discret (nombre entier d'entrées, nombre entier de propriétés) ${ }^{12}$ du système linguistique. La discrétisation est - en termes mathématiques ${ }^{13}$ - la représentation d'un système continu par des éléments discrets. La discrétisation est un passage à un nombre fini de données du système linguistique à partir de la prise en considération de toutes les données. Par exemple, leur formalisation et leur traitement par ordinateur nécessitent une discrétisation. Les deux citations suivantes apportent d'autres éclairages :

En mathématiques appliquées, la discrétisation est la transposition d'un état continu (fonction, modèle, variable, équation) en un équivalent discret. Ce procédé constitue en général une étape préliminaire à la résolution numérique d'un problème ou sa programmation sur machine.

$[\ldots]$

En statistique et apprentissage machine, la discrétisation renvoie à la conversion de variables ou caractéristiques continues en variables ou caractéristiques discrètes nominales. (« Discrétisation », Wikipédia $)^{14}$

Le LG fournit des descriptions beaucoup plus précises que dans la pratique habituelle, parce qu'elles prennent en compte systématiquement tout le vocabulaire et un jeu abondant de constructions syntaxiques, et parce qu'elles en détaillent l'acceptabilité ou l'inacceptabilité. En compensation, il modère ses ambitions d'un autre côté, car on décrit bien l'acceptabilité des structures lexico-syntaxiques pour une langue, mais on ne le fait pas :

- pour chaque locuteur, parce ce que ça serait un travail excessif. En revanche, les variantes dont ce locuteur unique peut être porteur font l'intérêt de la sociolinguistique et du CL;

- pour un état de langue antérieur, parce qu'on n'a plus les locuteurs pour fournir les inacceptabilités nécessaires. En revanche, les variantes historiques font l'intérêt de la sociolinguistique et du CL.

La sociolinguistique ambitionne d'aborder la covariance des phénomènes linguistiques et sociaux en étudiant les uns à travers les autres, considérés tour à tour comme des causes et des effets. Elle procède à partir de corpus produits en un temps, en un lieu, en un milieu déterminés, afin d'étudier ce qui varie ou a changé (ou ce qui ne varie pas ou n'a pas changé).

La sociolinguistique modère ses ambitions d'un autre côté car ses études sont vouées à se limiter à un échantillon d'entrées lexicales ou à un échantillon de constructions syntaxiques, ou aux deux, sous peine de réduire leur prise en compte

\footnotetext{
${ }^{12}$ Bien entendu, il existe des modèles tout à fait différents d'analyse linguistique, par exemple celui de VICTORRI (2004) qui adopte un modèle continu en sémantique lexicale.

${ }^{13}$ MAWHIN (2017) cite Delahaye et Rechenmann là où ils soulignent avec pertinence que « La question est, non pas de savoir si un modèle est correct ou non, mais d'apprécier son apport dans un processus de compréhension progressive du système et de ses comportements, motivé par un ensemble de questions initialement explicitées. Un bon modèle est un modèle utile ».

${ }^{14}$ Cf. https://fr.wikipedia.org/wiki/Discrétisation (dernière consultation : 22/07/2021). 
de la diversité sociale ou individuelle, ou de se heurter à des acceptabilités indécidables.

Concernant le CL, comme le prévoit la théorie depuis Saussure, c'est en comparant deux descriptions de deux états de langues qu'on peut observer une évolution. Ainsi, le chercheur en CL explore les corpus disponibles de textes produits à des états antérieurs de la langue étudiée, ou bien il construit des corpus sur mesure pour les consulter. Nous avons vu que les corpus ne sont que des échantillons ; ces descriptions ne sont donc pas fondées en extension sur la langue, comme le sont les descriptions du LG, qui utilise désormais abondamment les corpus, mais compense leur finitude en recourant à l'introspection pour juger l'acceptabilité des combinaisons entrée lexicale / construction syntaxique quand elles ne sont pas représentées de façon statistiquement significative dans le corpus.

Le chercheur en CL peut exploiter les descriptions du LG, mais seulement celles qui ont été effectuées à partir des années 1960, qui marquent son début. Comment faire pour les états antérieurs ou plus anciens?

Des lexico-grammairiens ont commencé à réaliser des descriptions systématiques et précises de certains faits de langue à partir de certains textes anciens. Leurs études ont été effectuées sur des corpus limités et elles ne permettent donc pas à présent de généraliser les résultats obtenus. Néanmoins, la précision du LG, supérieure à celle obtenue par d'autres études, permet de tirer des résultats plus pertinents.

Nous allons fournir plusieurs exemples de CL mis en lumière grâce au LG.

1. Examinons le cas de faits de langue relatifs à un état antérieur qui n'ont pas été conservés. À ce propos, il existe des études menées sur les verbes supports en portugais ancien (CHACOTO, 1997 et 1998 ; RANCHHOD, 1999 ; PANTE \& ORTEGA, 2009 ; PANTE, 2012 ; CALLOU, 2006 ; entre autres). Rappelons qu'on doit la notion de support à Maurice Gross et que, appliquée aux verbes, elle indique ceux qui ne remplissent pas la fonction de prédicat ${ }^{15}$ d'une phrase simple, cette fonction étant assurée par un nom ou un adjectif, qu'on appellera donc nom (1-3) ou adjectif prédicatif (4) :

\section{(1) Max fait un voyage en Australie}

= (1a) Max voyage en Australie

(2) Max donne une gifle à Luc

= (2a) Max gifle Luc

(3) Max a du respect pour Marie

= (3a) Max respecte Marie

(4) Max est fidèle à ses origines

\footnotetext{
${ }^{15} \mathrm{Au}$ passage, en ce qui concerne la fonction « support » des verbes, on peut remarquer que celle-ci et la fonction prédicative d'une même forme verbale ne s'excluent pas automatiquement, comme le montre RADIMSKÝ (2010) qui décrit la relation entre forme (verbe fini) et fonction (support, prédicat) en termes de centre-périphérie, deux notions formulées au sein de l'École de Prague.
} 
Les chercheuses portugaises et brésiliennes ont dû faire face à des difficultés importantes. Leurs compétences linguistiques et extralinguistiques divergent de celles des locuteurs de la période prise en examen. De plus, il leur était exclu de se renseigner auprès d'autres locuteurs pour tester l'acceptabilité des données. Prenant en compte ces anomalies, elles ont dû se construire un corpus à partir de textes sélectionnés. Jusque-là, il s'agit de la démarche habituelle suivie par les linguistes.

Mais ces chercheuses ont travaillé en adoptant la méthode du LG, par laquelle il faut rechercher les faits de langue en extension dans le lexique et les décrire systématiquement avec précision. Elles ont donc mené un recensement complet des occurrences des diverses constructions nominales régies par des verbes. Elles ont choisi un verbe ou plusieurs (estar, fazer, haver, ser, ter, tomar, etc.) et ont étudié des occurrences de leurs combinaisons avec des noms ou des groupes nominaux. Elles ont compris leur statut de verbe plein, auxiliaire ou support, et trouvé des analogies et des différences par rapport au portugais actuel, même dans sa variante de portugais du Brésil. Finalement, elles ont essayé de tirer des conclusions sur la conservation (ou la disparition) de ces constructions de nos jours.

Or, les corpus construits pour ces recherches sont limités et ils ne permettent donc pas de faire des généralisations. L'étude des constructions Vsup Npréd en portugais médiéval devrait bien sûr se poursuivre. Il est donc nécessaire d'exploiter tout le corpus qui nous est parvenu pour illustrer plus de faits de la langue ancienne. Il reste encore de nombreuses questions à analyser.

Cependant, regardons quelques combinaisons Vsup Npréd que nous tirons de CHAсото (1998) et qui ne sont plus employées en portugais de nos jours. La première porte sur dar torvam (« tonner ») :

(5) E logo depois que o torvam deu Et juste après que le tonnerre a donné (Et juste après que le tonnerre a éclaté) ${ }^{16}$

La deuxième combinaison porte sur fazer ajuda («donner de l'aide ») :

(6) nom sodes vos teudo de lhe fazerdes ajuda de todo vosso poder? n'êtes vous [pas] tenu de lui faire aide de tout votre pouvoir? (ne devez-vous pas lui donner de l'aide autant que vous le pouvez ?)

La troisième combinaison comporte toujours le verbe faire dans fazer amor (« faire l'amour ») :

(7) el era cavaleiro do mundo mais amado e que mais amor e onrra fazia aos cavaleiros il était le chevalier du monde le plus aimé et qui le plus d'amour et d'honneur faisait aux chevaliers

\footnotetext{
${ }^{16}$ En général, nous indiquons en caractères normaux la traduction littérale au deuxième niveau et l'équivalent sémantique, entre parenthèses, au troisième. Les notations employées sont celles du LG. 
(il était le chevalier le plus aimé du monde et celui qui aimait et honorait le plus les autres chevaliers)

Suivant CНАСото (1998: 130-131), nous remarquons que le mot amor à l'époque ne servait pas à exprimer le sens que Grevisse définit par «passion charnelle »(GREVISSE \& GOOSSE, 2016: § 471 a)), mais tous les autres (par exemple «affection», «attachement», etc.). La combinaison «faire l'amour» n'exprimait donc pas le fait d'avoir des relations sexuelles, comme aujourd'hui.

Nous remarquons également que, contrairement à l'usage actuel en portugais, la combinaison donne lieu à une séquence renversée ; dans (7), amor est suivi et non pas précédé de fazia, ce qui était possible dans l'état ancien de la langue et ne l'est plus à présent.

Par ailleurs, un autre fait syntaxique évident de CL réside dans la préposition qui suit fazer amor; c'est $a$ (《à ») qui a été remplacé par com («avec »).

L'inversion entre le verbe support et le syntagme nominal qui le complète est confirmée par l'étude de PANTE \& ORTEGA (2009) sur le verbe tomar, comme l'une des caractéristiques syntaxiques du CL en portugais. Nous donnons l'exemple suivant emprunté aux deux auteures (2009: 147) :

(8) E quanto ao primeiro erro, porque todo nace da voontade, a qual determyna nom querer encontrar, com receo que dello toma, reguardem o que screvy das cousas que o fazem perder, e ajudemse daquellas em que sentirem pera isto mais proveyto.

Et quant à la première erreur, puisque tout naît de la volonté, laquelle détermine [à] ne pas vouloir [la] rencontrer, avec l'appréhension qu'on en prend, regardez ce que j'ai écrit des choses qui la font perdre, et aidez-vous de celles dans lesquelles vous trouvez pour cela le plus de profit.

(Et quant à la première erreur, puisque tout découle de la volonté qui fait ne pas vouloir la commettre, à cause de l'appréhension qu'on en a, lisez ce que j'ai écrit sur les choses qui y font renoncer, et aidez-vous avec celles que vous trouvez les plus bénéfiques pour cela.)

En tout cas, СНАСОтО (1998 : 136) montre que parmi les 97 noms de son corpus qui sont construits avec faire, seulement quarante continuent à accepter ce verbe support. Ce qui équivaut, outre les exemples ci-dessus, à 57 cas de CL, soit $59 \%$ de CL.

2. Examinons le cas de vestiges d'états antérieurs dans un état synchronique, à savoir les vestiges que la langue conserve encore aujourd'hui ; il s'agit bien d'un cas particulier de l'évolution.

Tout d'abord, et pour en conclure avec le portugais, les études susmentionnées ont démontré que les constructions à verbe support et substantif prédicatif, très fréquentes de nos jours, ont été largement employées dans certains textes portugais des $\mathrm{XIV}^{\mathrm{e}}$ et $\mathrm{XV}^{\mathrm{e}}$ siècles; il existe donc de bonnes raisons pour estimer que ces constructions font partie de l'héritage de la langue portugaise. Voilà donc que la précision du LG permet une conclusion féconde à un niveau syntaxique de la réflexion linguistique.

En ce qui concerne le français, on peut trouver bien des exemples de l'évolution dans les tables du LG. 
2a. Examinons le cas, traditionnel, du changement de l'ordre des constituants. Cet ordre en latin, bien qu'il soit assez libre, est principalement SOV (sujet-objetverbe, c'est-à-dire non marqué, construit à gauche), tandis que les langues romanes sont principalement du type SVO (marqué, construit à droite).

Cependant, bien que le français ait généralement l'ordre SVO, cette typologie ne s'applique pas systématiquement, surtout avec les pronoms ${ }^{17}$ :

$$
\begin{aligned}
& \text { (9) Max adore ses enfants [SVO] } \\
& \text { (9a) Max les adore }
\end{aligned}
$$

Le LG enregistre la forme verbale adorer et ses propriétés dans deux tables, codées 12 et $32 \mathrm{r} 2^{18}$.

Dans la table 12, version imprimée (GROSS, 1975 : 365), à la ligne « adorer » on trouve un «+» dans la colonne «Complément direct / Noms / ppv», qui doit correspondre à :

(10) Max adore que ses enfants soient ce qu'ils sont

= (10a) Max adore ses enfants

$=(10 \mathrm{~b})$ Max les adore

Dans la table 12 en version électronique, en ligne ${ }^{19}$, cette propriété a pour intitulé «N1 = Ppv ». Dans la table 32r2, la propriété n'est pas représentée, parce qu'elle est vérifiée pour toutes les entrées, dans ce cas :

(11) Max adore les veaux d'or

= (11a) Max les adore

\footnotetext{
${ }^{17}$ Avec les subordonnées, en français on trouve également l'ordre OVS : Max adore ce qu'a fait untel.

${ }^{18}$ Les conventions de notation sont celles des lexico-grammairiens. Cf. aussi le site Web du LIGM : http://infolingu.univ-mlv.fr/ (dernière consultation : 22/07/2021). Par exemple, dans les lignes qui suivent, «ppv » désigne un pronom préverbal.

${ }^{19}$ Le téléchargement du LG, sous licence LGPLLR, peut être effectué à partir du site Web du LIGM : http://infolingu.univ-mlv.fr/ (dernière consultation : 22/07/2021).
} 
Voici un extrait de la table 12, version imprimée (GROSS, 1975 : 365) :

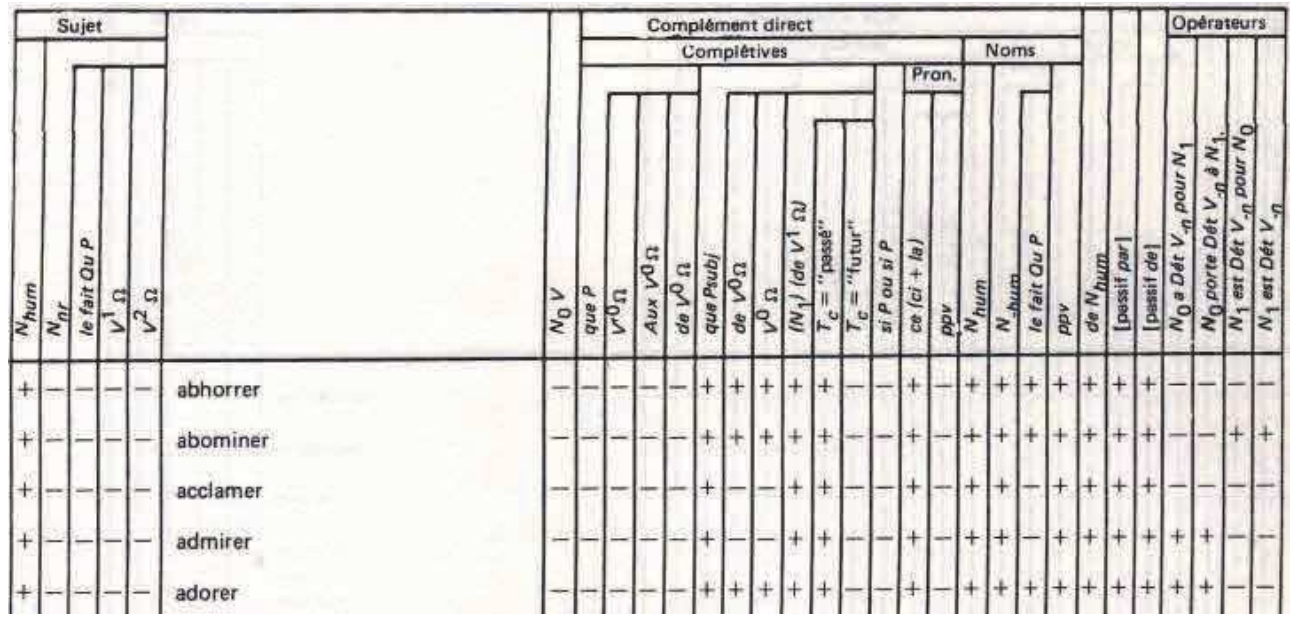

Fig. 3.

Un chercheur en CL qui aurait envie d'étudier l'ordre des constituants en français pourrait retrouver bien d'autres exemples dans le LG.

Quant à la dimension syntaxique du CL, qui nous intéresse spécialement, mentionnons une théorie du changement, la grammaticalisation (COMBETTES, 2008; BÉGUELIN, 2014). Un processus linguistique diachronique réduit progressivement la signification d'un mot lexical jusqu'à ce qu'il ne remplisse qu'une fonction grammaticale. Il s'agit par exemple du passage des structures latines $\operatorname{Adj} N$ où $N=:$ mente aux mots lexicaux français ayant fonction d'adverbes, construits avec le suffixe -ment et notés $A d v$-ment : du latin bonā mente $\rightarrow$ au français bonnement. Voici un extrait de la table PADV qui enregistre un type d'adverbes figés du français, dont certains en -ment (GROSS, 1986a : 318) :

\begin{tabular}{|c|c|c|c|c|c|}
\hline 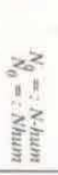 & & & 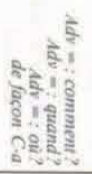 & 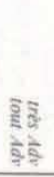 & 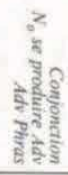 \\
\hline $\begin{array}{l}+- \\
+- \\
+- \\
+- \\
+- \\
+- \\
++ \\
++\end{array}$ & $\begin{array}{l}\text { tricher } \\
\text { être content } \\
\text { injurier N } \\
\text { transforme-toi ! } \\
\text { venir? } \\
\text { travailler } \\
\text { tricher } \\
\text { vexer Nhum } \\
\text { surprendre Nhum }\end{array}$ & $\begin{array}{l}\text { "Dnum-ièmement" } \\
\text { "n-uplement" } \\
\text { abondamment } \\
\text { abracadabra ! } \\
\text { absolument ! } \\
\text { actuellement } \\
\text { additionnellement } \\
\text { affreusement } \\
\text { agréablement }\end{array}$ & 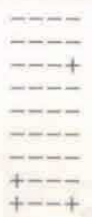 & $\begin{array}{l}-- \\
-\overline{-} \\
-+ \\
-- \\
\overline{--} \\
\overline{-} \\
-+\end{array}$ & 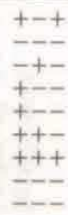 \\
\hline
\end{tabular}

Fig. 4.

En passant, le changement de l'ordre des constituants évoque, entre autres, les variations de l'ordre logique, qui constitue un vrai mythe du français : 
L'ordonnateur du génie de la langue française est le principe supposé que l'ordre des mots dans la phrase est l'ordre sujet-verbe-complément; que cet ordre caractérise le français à la différence des autres langues; qu'il est le seul ordre logique, celui de la raison naturelle. Par lui le français est la langue même de la raison. (MESCHONNIC, 1997 : 161)

En fait, dans cette citation, nous avons affaire à l'ordre des mots et à l'ordre logique. Considérons l'exemple suivant, que nous empruntons à GROSS (1989 : 10) :

(12) Les enfants (adorent + aiment) le théâtre

En ce qui concerne l'ordre des mots, la phrase en (12) montre en effet l'ordre sujet-verbe-complément. Cependant, en français on peut également observer des phrases d'ordre inverse et les contre-exemples à cette règle sont surtout les phrases interrogatives :

(12a) Qu'(adorent + aiment) les enfants ?

En ce qui concerne l'ordre logique, la phrase en (12) montre un ordre où un verbe place un argument humain à gauche et un argument non-humain à droite. Maurice GROSS (1989: 10) remarque qu'en français on peut également observer des phrases d'ordre inverse :

(12b) Le théâtre (intéresse + passionne) les enfants ${ }^{20}$

En outre,

il existe aujourd'hui moins de 100 verbes du type aimer et plus de 600 du type intéresser. Qui plus est, la création de vocabulaire (e.g. argot de lycée) se fait sur le type intéresser (e.g. brancher, câbler). (GROSS, 1989: 10)

Maurice Gross conteste que l'ordre de (12) soit le seul logique et appartienne à la raison naturelle. En effet, (12b) ordonne les mêmes arguments que (12) mais à l'envers, donc selon une logique inverse, et les verbes du français ordonnant les arguments comme en (12b) sont plus nombreux que les verbes les ordonnant comme en (12). En quoi (12b) serait-il moins logique que (12), ou le serait-il plus ?

Si l'ordre qui caractérise le français est le seul conforme à la logique et à la raison naturelle, celles-ci exigent donc, pour le même message à transmettre, tantôt un ordre, et tantôt l'inverse de cet ordre. Mais alors, si elles sont si larges d'esprit, pourquoi n'admettent-elles pas aussi l'ordre observé dans les langues autres que le français?

\footnotetext{
${ }^{20}$ Les exemples (12) vs (12b) pourraient être analysés autrement selon une perspective fonctionnelle de l'énoncé (ou «topic-focus articulation»); cf. le numéro thématique 1/2012 de l'Écho des études romanes édité par RADIMSKÝ, qui a été entièrement consacré à la question de la perspective fonctionnelle de la phrase au moyen de l'apport du Cercle de Prague (https://www.eer.cz/?s=2012_1, dernière consultation: 22/07/2021), ainsi qu'un article sur les perspectives fonctionnelles des constructions à verbe support (RADIMSKÝ, 2013).
} 
Le LG rend compte aussi de l'ordre des arguments et le chercheur du CL peut interroger le LG (en ligne) pour compter les verbes qui acceptent l'ordre inverse et en estimer l'importance.

2b. Examinons le cas du changement de la catégorisation des données linguistiques, soit la nominalisation des infinitifs qu'on trouve dans la langue littéraire alors qu'elle est peu employée dans la langue commune. GREVISSE \& GoOSSE (2016: § 197) en donnent plusieurs exemples, dont le complément adverbial au sortir de contenant l'infinitif nominalisé sortir et vérifié chez Zola. Or ce complément est analysable comme adverbe figé en LG. Il est en effet enregistré dans la table PCDN (GROSS, 1986a : 468-469) :

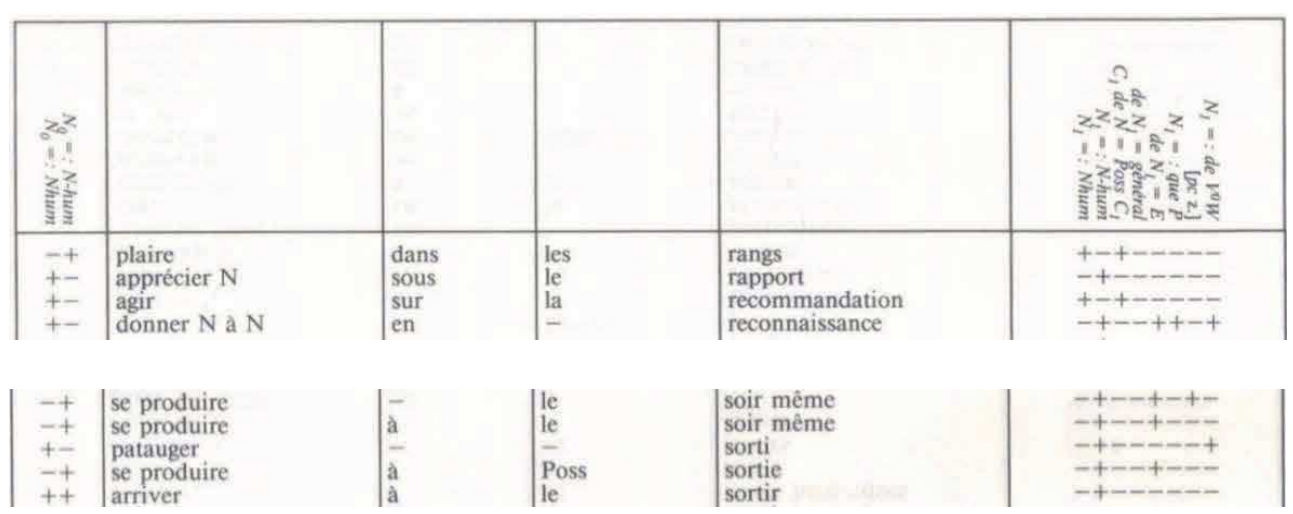

Fig. 5.

2c. Examinons le cas du changement de la construction syntaxique des données linguistiques, soit le gérondif construit à l'aide d'autres prépositions alternatives. L'exemple de l'adverbe figé à son corps défendant témoigne d' « un état de langue très ancien où le gérondif pouvait être introduit indifféremment par les prépositions à, en, pour, sans. » (WAGNER \& PINCHON, 1962 : 323). Le LG du français enregistre cet adverbe dans la table PCA (GROSS, 1986a : 400-401) :

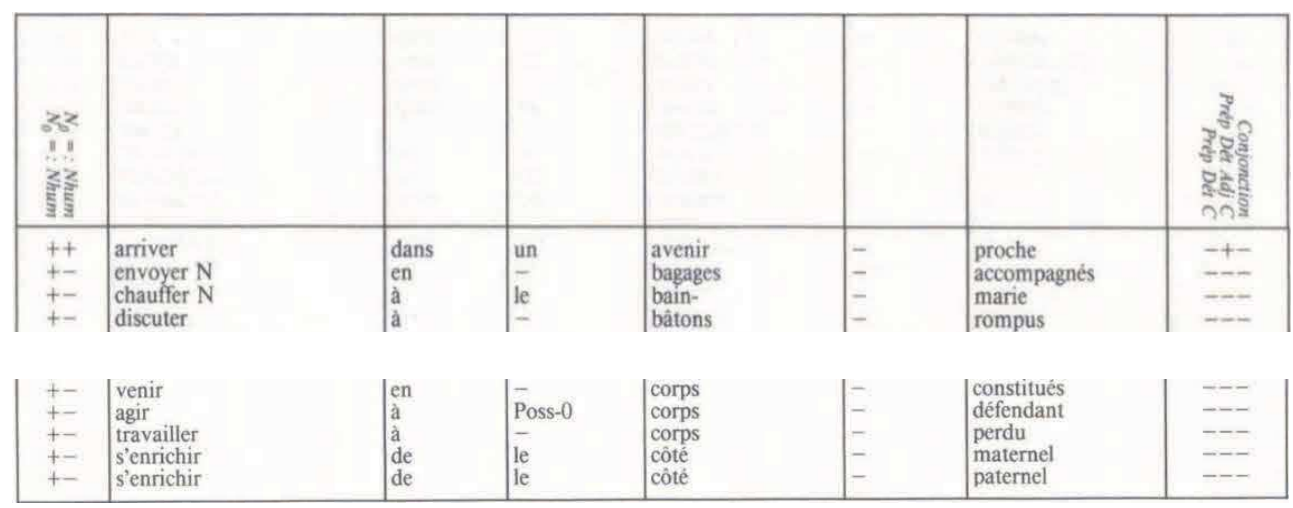

Fig. 6. 
Le classement lexico-grammatical des deux adverbes figés au sortir de et à son corps défendant montre que "dans de nombreuses descriptions synchroniques, il s'introduit des formes qui ont souvent une interprétation diachronique naturelle. » (GROSS, 1975 : 227). En effet,

Un état de langue comporte simultanément des niveaux diachroniques et dialectaux variés. Par exemple, le français contemporain dit cultivé ou littéraire est vraisemblablement constitué d'une part importante de français classique. (GRoss, 1975 : 228)

La distinction entre données synchroniques et diachroniques est strictement pratique, et non théorique. En effet, il n'y a aucune différence essentielle entre un système linguistique vivant à une date et un autre vivant à une autre date. La seule différence est que si le système n'est plus vivant, nous n'avons plus accès directement aux locuteurs, ce qui est une différence méthodologique, «pratique ».

2d. Examinons le cas du changement de la combinaison lexico-syntaxique dû aux variétés de français. Il existe en effet des liens entre variation dialectale et CL.

Une bonne part de ce qui différencie deux états diachroniques d'une langue peut se refléter dans deux variétés contemporaines de cette langue. (LYONS, 1970 : 40)

En LG, il existe des descriptions exhaustives comparées de langues et de variétés diverses. Parmi les multiples références bibliographiques ${ }^{21}$, nous citons le projet BFQS de construction d'un Lexique-Grammaire Comparé - noté dorénavant LGC - des expressions verbales figées des français de Belgique, de France, du Québec, de Suisse (LAMIROY et al., 2010). Voici un extrait de la table A, disponible en ligne ${ }^{22}$ :

\footnotetext{
${ }^{21}$ Pour la bibliographie du LG, cf. le site Web du LIGM : http://infolingu.univ-mlv.fr/ (dernière consultation : 22/07/2021).

${ }^{22}$ La table A est disponible en ligne sur le site Web du CENTAL : https://uclouvain.be/fr/institutsrecherche/ilc/cental/les-expressions-verbales-figees-bfqs.html (dernière consultation : 17/04/2020). 


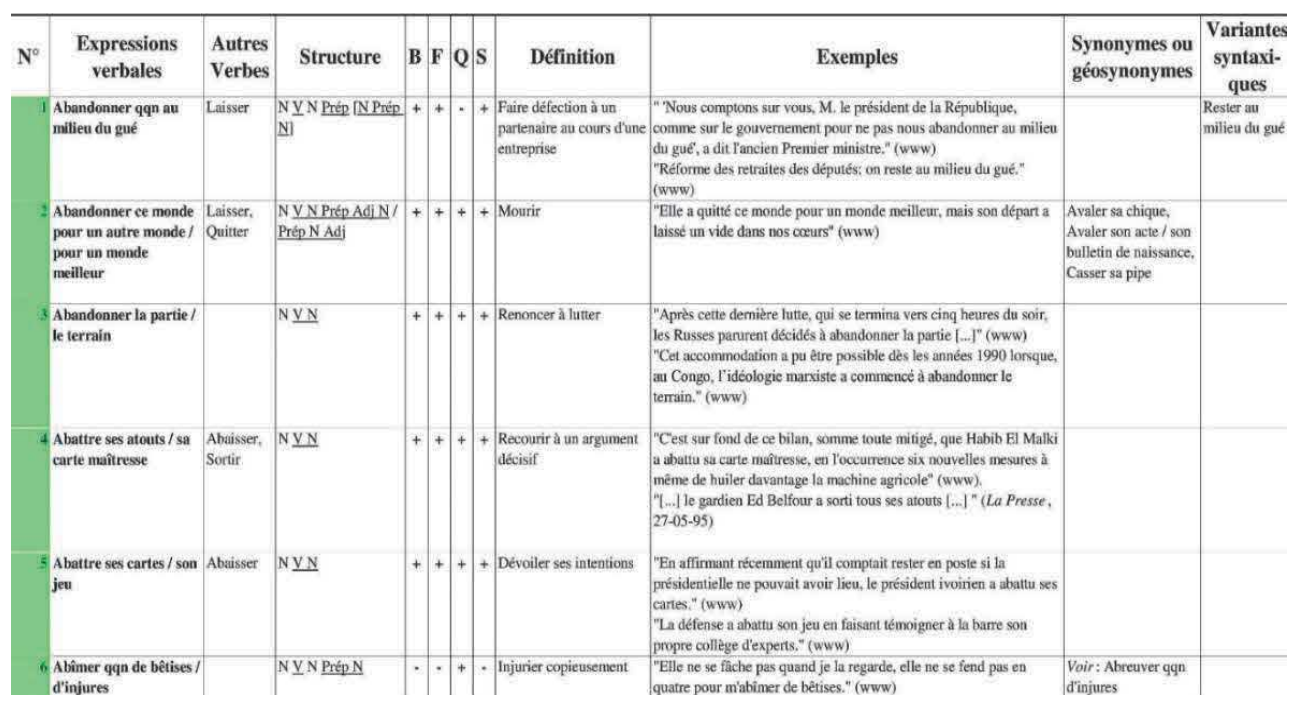

Fig. 7.

Un aspect important à préciser est que, si dans $\mathrm{BFQS}$ on trouve l'indication de données appartenant aussi au français commun à deux ou plusieurs variétés, leur description est fondée sur des LG différentiels, qui n'enregistrent que des données propres à chaque variété. La comparaison rigoureuse et la formalisation stricte des faits linguistiques nous permettent ainsi de reconsidérer d'un point de vue technique les notions de norme et de variétés linguistiques.

Nous mentionnons également notre participation au projet de construction du LGC des langues romanes, avec deux volumes et de nombreux articles consacrés à la comparaison et à la traduction des adverbes figés principalement du français et de l'italien, mais aussi du québécois, de l'espagnol, du portugais, de l'anglais. En ce qui concerne encore l'Amérique du nord, nous donnons un extrait d'un lexique bilingue italien-québécois (DE GIOIA, 2004 : 163) :
(PAC) partir à bonne heure
(PAC) partire di buon'ora
(PAC) partire di buon mattino
(PDETC) partire al mattino
(PCPC) partire di mattina presto
(PAC) partir à la fine course
(PVCO) partire come una palla di schioppo
(PVCO) partire come un razzo
(PCONJ) partire in men che non si dica
(PCONJ) partire in quattro e quattr'otto
$(\mathrm{PCONJ})$ partire a remo e vela
(PCONJ) partire in fretta e furia
(PCDC) partire in meno di un amen
(PCDC) partire in meno di un ave
(PCDC) partire in meno di un pater
(PCDC) partire in meno di un secondo
(PDETC) partire in un attimo 
(PDETC) partire in un amen

(PDETC) partire in un ave

(PDETC) partire in un pater

(PDETC) partire in un secondo

(PDETC) partire in tutta fretta

(PAC) partir au plus christ $\quad$ (PCA) partire il più presto possibile (PAC) partire al più presto

Fig. 8.

Les fig. 7 et 8 montrent des classifications concernant le phénomène du $\mathrm{CL} /$ variation. Elles sont manifestement inspirées des premières tables du LG (fig. 3). Par conséquent, elles peuvent être considérées par les chercheurs en CL comme une actualisation du codage binaire de l'acceptabilité qui, aux origines du LG, leur semblait laisser peu d'espace pour rendre compte de la variation (socio)linguistique. De nos jours, la méthode du LG est bien applicable à l'analyse du phénomène, sans porter préjudice à l'exigence de fournir des données formalisées et discrètes.

2e. Pour replacer tout cela dans le contexte du rapport entre CL et LG, nous avons montré comment et pourquoi le LG décrit et représente le changement.

Tout ce qui précède atteste donc que les chercheurs en CL peuvent profiter des acquis du LG que nous avons expliqués. Mais il y a d'autres perspectives et pistes de recherches qui s'ouvrent, dont celle, qui est à notre avis prometteuse, d'une collaboration nouvelle entre le sociolinguiste et le lexico-grammairien.

À propos du sociolinguiste, de nos jours il explore de plus en plus de grands corpus, mis au point principalement dans le domaine de l'oralité. Et comme nous l'avons vu plus haut, les grands corpus oraux donnent certes des informations sur la diversité sociologique, mais leur utilisation exclusive limite la couverture lexicale et grammaticale. Le sociolinguiste pourrait exploiter le LG en tant que grand corpus de tables syntaxiques validées et bien structurées.

Quant au lexico-grammairien, il faut rappeler que, au cours des deux premières décennies d'études lexico-grammaticales, à savoir les années 60 et $70 \mathrm{du} \mathrm{XX}{ }^{\mathrm{e}}$ siècle, les auteurs du LG ont pris des décisions d'acceptabilité des données en interrogeant un nombre assez limité de locuteurs. Ces derniers étaient très souvent des linguistes appartenant à l'équipe internationale qui collaborait avec le $\mathrm{LADL}^{23}$. Il serait intéressant d'étendre les tests de jugement d'acceptabilité à des locuteurs ayant d'autres compétences, pour peaufiner les descriptions lexico-grammaticales. Ainsi Labelle, qui a intégré dans le LG la variété de français du Québec, affirme que :

Les études sur le terrain, jusqu'à maintenant, ont été surtout effectuées par les sociolinguistes, qui n'ont pas abordé les mêmes problèmes. Il est important de mettre au point une méthode qui,

\footnotetext{
${ }^{23}$ Le Laboratoire d'Automatique Documentaire et Linguistique (CNRS et Université Paris 7) fut fondé et dirigé par Maurice Gross. Le LADL prenait d'une certaine façon, en 1970, la suite de la Section d'automatique documentaire (SAD), qui existait depuis 1961 et avait été dirigée par Jean-Claude Gardin jusqu'en 1966 puis par Maurice Gross (LÉON, 1998 et 2015).
} 
dans le cadre du LG, permette de mettre en lumière certaines données reliées à la notion de compétence et à celle de corpus. (LABELLE, $1995: 22$ )

C'est justement dans la mise au point d'une nouvelle méthode de validation, plus large, des données déjà recueillies, décrites et classées, que pourrait se réaliser une piste de recherche féconde. Il en dériverait le défi de maîtriser la quantité de données, ce qui présupposerait un compromis entre la couverture lexicale, la couverture grammaticale et la couverture sociolinguistique. On ne peut pas tout avoir. Par exemple, on pourrait définir un grand corpus original, dont les informations à enregistrer seraient choisies par un comité de spécialistes en CL et en LG.

\section{Pour conclure}

Nous avons étudié la problématique du rapport entre les deux domaines de recherche du changement linguistique et du Lexique-Grammaire. Pour le premier domaine, nous avons approfondi les notions linguistiques de "changement» et «variation», et indiqué comment elles débouchent sur l'oralité. Pour le second domaine, nous avons présenté la méthode et précisé certains principes fondamentaux; nous avons ensuite montré plusieurs descriptions lexicogrammaticales qui enregistrent des phénomènes intéressants de changement linguistique. Enfin, après avoir cadré théoriquement ces domaines et mis en valeur des possibilités de contact, nous avons également suggéré des perspectives de recherche collaborative.

\section{BIBLIOGRAPHIE}

ARMSTRONG Nigel (2008), Les Français devant la 'norme urbaine non méridionale'. Changement linguistique et identité sociale, Cahiers de sociolinguistique 13, p. 15-29.

ARRIVÉ Michel, GADET Françoise, GALMICHE Michel (1986), La grammaire d'aujourd'hui: guide alphabétique de linguistique française, Paris, Flammarion.

BAUDE Olivier (dir.) (2006), Corpus oraux, guide des bonnes pratiques, Paris/Orléans, Éditions du CNRS / PU d'Orléans.

BÉGUELIN Marie-José (2014), Deux points de vue sur le changement linguistique, Langages 196, p. 13-36.

BERRUTO Gaetano (2017), System-oriented and speaker-oriented approaches in Italian sociolinguistics, Sociolinguistic Studies 11 (2-4), p. 271-290.

BLANCHE-BENVENISTE Claire (1997), La notion de variation syntaxique dans la langue parlée, Langue française 115, p. 19-29.

Blanche-Benveniste Claire, JEAnJean Colette (1986), Le français parlé, transcription et édition, Paris, Didier-Érudition.

CALlou Dinah (2006), Variação e mudança lingüísticas : a substituição de haver por ter, in: SEDANo Mercedes, Bolívar Adriana, SHIRo Martha (coord.), Haciendo Lingüística. Homenaje a Paola Bentivoglio, Caracas, Comisión de 
Estudios de Postgrado, Facultad de Humanidades y Educación, Universidad Central de Venezuela, Colección Textos y Documentos, p. 147-161.

CAPPEAU Paul, GADET Françoise (2007), L'exploitation sociolinguistique des grands corpus. Maître-mot et pierre philosophale, Revue française de linguistique appliquée 12 (1), p. 99-110.

CHAcoto Lucília (1997), Predicados nominais com fazer no português medieval, in: CAstro Ivo (éd.), Actas do XI Encontro Nacional da Associação Portuguesa de Linguística, Lisboa, Colibri, vol. II, p. 69-77.

Снасото Lucília (1998), Les noms prédicatifs construits avec fazer (faire) au Moyen Âge, Cahiers de l'Institut Linguistique de Louvain 24 (3-4), p. 127-144.

COHEN Marcel (1950), Le langage : structure et évolution, Paris, Éditions sociales.

COMBETTES Bernard (2008), Théories du changement et variations linguistiques : la grammaticalisation, Pratiques 137-138, p. 135-145.

DE GIOIA Michele (2004), Un lexique bilingue québécois-italien des adverbes figés, in : Dotoli Giovanni (éd.), Atti del Seminario di Studi canadesi "I colori del Canada", Pescara, 28-29 novembre 2003, Fasano, Schena, p. 149-164.

DE GIOIA Michele (2015), Le Lexique-Grammaire. Introduction à la méthode, in : PAisSa Paola, Rigat Françoise, VitToz Marie-Berthe (éd.), Dans l'amour des mots. Chorale(s) pour Mariagrazia, Alessandria, Edizioni dell'Orso, p. 299309.

DeBAisieuX Jeanne-Marie, BERTIN Tiphanie, HusianyCiA Magali (2008), Corpus Oraux : recueil et analyse de données - Introduction, Verbum 30 (4), p. 245 259.

Delaveau Annie, Huot Hélène, Kerleroux Françoise (1972), Questions sur le changement linguistique, Langue française 15, p. 29-46.

DEULOFEU Henri-José (2017), Les constructions comme variables pour la variation syntaxique et le rôle des traditions discursives dans la production des variantes, Revue de Sémantique et Pragmatique 41-42, p. 117-134.

Dubois Jean, MATHÉE Giacomo, GUESPIN Louis, MARCELleSI Christiane, MARCELlesi Jean-Baptiste, MÉVEL Jean-Pierre (2002), Dictionnaire de linguistique, Paris, Larousse.

GADET Françoise (dir.) (1997), La variation en syntaxe, Langue française 115.

GADET Françoise (2003), La variation sociale en français, Paris, Ophrys, coll. L'essentiel.

GADET Françoise (2006), La langue et le sociolinguistique, Synergies France 5, Les enjeux sociaux du langage. Hommage à Bernard Gardin, p. 81-86.

GADET Françoise (2020), Langue et variation, in : Encyclopédie grammaticale du français, en ligne : http://encyclogram.fr (dernière consultation : 22/07/2021).

GrevisSE Maurice, Goosse André (2016), Le bon usage, 16éd., Louvain-laNeuve, De Boeck Supérieur.

Gross Maurice (1968), Grammaire transformationnelle du français. 1 : Syntaxe du verbe, Paris, Larousse (réédité sous le même titre : Paris, Cantilène, 1986).

Gross Maurice (1975), Méthodes en syntaxe, Paris, Hermann.

GROSS Maurice (1977), Grammaire transformationnelle du français. 2 : Syntaxe du nom, Paris, Larousse (réédité sous le même titre : Paris, Cantilène, 1986). 
Gross Maurice (1986a), Grammaire transformationnelle du français. 3 : Syntaxe de l'adverbe, Paris, ASSTRIL.

GROSS Maurice (1986b), Lexique-grammaire et adverbes : deux exemples, Revue Québécoise de Linguistique 15 (2), p. 299-311.

Gross Maurice (1989), Préface, in : La lingua francese nel seicento, Bari/Paris, Adriatica/Nizet, p. 9-10.

HARRIS Zellig Sabbetai (1962), String Analysis of Sentence Structure, The Hague, Mouton.

HARRIS Zellig Sabbetai (1976), Notes du cours de syntaxe, traduction française par Gross Maurice, Paris, Le Seuil.

HARRIS Zellig Sabbetai (2007), La langue et l'information, traduction française par IBRAHIM Amr Helmy, MARTINOT Claire, Paris, CRL.

LABELLE Jacques (1995), Lexique-grammaire et variation en français, in : LABELLE Jacques, LECLÈRE Christian (éd.), Lexiques-grammaires comparés en français, Lingvistica Investigationes Supplementa 17, Amsterdam/Philadelphia, John Benjamins, p. 13-28.

LABOV William (1994), Principles of Linguistic Change. 1: Internal Factors, Oxford, Basil Blackwell.

LABOV William (2001), Principles of Linguistic change. 2 : Social Factors, Oxford, Blackwell.

LABOV William (2010), Principles of Linguistic change. 3 : Cognitive and Cultural Factors, Oxford, Wiley Blackwell.

LAMIROY Béatrice, KleIN Jean-René, LABELlE Jacques, LeClÈre Christian, MEUNIER Annie, ROSSARI Corinne (2010), Les expressions verbales figées de la francophonie: Belgique, France, Québec et Suisse, Paris, Ophrys, coll. L'essentiel.

LAZARD Gilbert (2007), Mathesius lu à la lumière de Saussure, Écho des études romanes III (1-2), p. 13-23.

LeCointre Simone, Le Galliot Jean (1973), Le changement linguistique : problématiques nouvelles, Langages 32, p. 7-26.

LEDEGEN Gudrun, LÉGLISE Isabelle (2013), Variations et changements linguistiques, in: SIMONIN Jacky, WhARTON Sylvie (dir.), Sociolinguistique des langues en contact, Paris, ENS, p. 315-329.

LÉON Jacqueline (1998), Les débuts de la traduction automatique en France (19591968) : à contretemps ?, Modèles Linguistiques 19 (2), p. 55-86.

LÉON Jacqueline (2015), Histoire de l'automatisation des sciences du langage, Lyon, ENS.

LYONS John (1970), Linguistique générale. Introduction à la linguistique théorique, traduction française par DUBOIS-CHARLIER Françoise, ROBINSON David, Paris, Larousse, coll. Langue et langage.

MARTINET André (1975), Évolution des langues et reconstruction, Paris, PUF.

MARTINET André (1955), Économie des changements phonétiques, in : Traité de phonologie diachronique, Berne, Francke.

MATHESIUS Vilém (1911), O potenciálnosti jevů jazykových [On the Potentiality of the Phenomena of Language]. Věstník Královské české společnosti nauk, 
1911-12, třída filozoficko-historickojazykozpytná, č. 2, únor 1911, p. 1-24 [traduit par VACHEK Josef (compiled by) (1964)].

MAWHIN Jean (2017), Les modèles mathématiques sont-ils des modèles à suivre?, Bruxelles, Académie royale de Belgique, coll. L'Académie en poche 97.

MESCHONNIC Henri (1997), De la langue française. Essai sur une clarté obscure, Paris, Hachette.

PANTE Maria Regina (2012), O verbo tomar como verbo-suporte no português arcaico, Linguas \& Letras 13 (24) (I ${ }^{\circ}$ Sem. 2012), p. 161-175.

PANTE Maria Regina, ORTEGA Érica Fernanda (2009), O verbo tomar como verbosuporte no Livro da Ensinança de Bem Cavalgar Toda Sela, Revista Philologus 15 (43) (jan./abr. 2009), p. 144-152.

RANCHHOD Elisabete Marques (1999), Construções com nomes predicativos na Crónica Geral de Espanha de 1344, in : FARIA Isabel Hub (éd.), Lindley Cintra. Homenagem ao Homem, ao Mestre e ao Cidadão, Lisboa, Cosmos, p. 667-681.

RADIMSKÝ Jan (éd.) (2007), Synchronie dynamique du système linguistique, Écho des études romanes III (1-2).

RADIMSKÝ Jan (2007), De la potentialité à la synchronie dynamique : un héritage oublié du Cercle de Prague, Écho des études romanes III (1-2), p. 7-12.

RADIMSKÝ Jan (2010), Supports centraux et supports périphériques : des « maillons faibles » du Lexique-Grammaire ?, Écho des études romanes VI (1-2), p. 181193.

RADIMSKÝ Jan (éd.) (2012), Perspective fonctionnelle de la phrase - l'apport du Cercle de Prague, Écho des études romanes VIII (1).

RADIMSKÝ Jan (2013), Quelles sont les 'perspectives fonctionnelles' de la prédication analytique? La structure thématico-rhématique des constructions à verbe support, Verbum $X X X V(1-2)$, p. 107-118.

SCHMIDT Thomas (2010), EXMARaLDA : un système pour la constitution et l'exploitation de corpus oraux, in : BOYER Henri (dir.), Pour une épistémologie de la sociolinguistique. Actes du colloque international de Montpellier 10-12 décembre 2009, Limoges, Lambert-Lucas, p. 319-327.

VACHEK Josef (compiled by) (1964), A Prague School Reader in Linguistics, Bloomington, Indiana university press.

Victorri Bernard (2004), Continu et discret en sémantique lexicale, Cahiers de praxématique 42 , p. $75-94$.

WAGNER Robert-Léon, PINCHON Jacqueline (1962), Grammaire du français classique et moderne, Paris, Hachette.

WeINREICH Uriel, LABOV William, HeRzoG Marvin I. (1968), Empirical foundations for a theory of language change, in: LEHMANN Winfred P., MALKIEL Yakov (éd.), Directions for historical linguistics, Austin, University of Texas Press, p. 95-188.

WISSNER Inka (2012), Les grands corpus du français moderne : des outils pour étudier le lexique diatopiquement marqué ?, SKY Journal of Linguistics 25, p. 233-272. 